\title{
A Disconnection Account of Gerstmann Syndrome: Functional Neuroanatomy Evidence
}

\author{
Elena Rusconi, PhD, ${ }^{1,2}$ Philippe Pinel, PhD, ${ }^{1,3,4}$ Evelyn Eger, MD, ${ }^{1,3,4}$ Denis LeBihan, MD, PhD, ${ }^{3,4}$ \\ Bertrand Thirion, $\mathrm{PhD},{ }^{3}$ Stanislas Dehaene, $\mathrm{PhD},{ }^{1,3,4}$ and Andreas Kleinschmidt, MD, $\mathrm{PhD}^{1,3,4}$
}

\begin{abstract}
Objective: To examine the functional neuroanatomy that could account for pure Gerstmann syndrome, which is the selective association of acalculia, finger agnosia, left-right disorientation, and agraphia.

Methods: We used structural and functional neuroimaging at high spatial resolution in healthy subjects to seek a shared cortical substrate of the Grundstörung posited by Gerstmann, ie, a common functional denominator accounting for this clinical tetrad. We construed a functional activation paradigm that mirrors each of the four clinical deficits in Gerstmann syndrome and determined cortical activation patterns. We then applied fiber tracking to diffusion tensor images and used cortical activation foci in the four functional domains as seed regions.

Results: None of the subjects showed parietal overlap of cortical activation patterns from the four cognitive domains. In every subject, however, the parietal activation patterns across all four domains consistently connected to a small region of subcortical parietal white matter at a location that is congruent with the lesion in a well-documented case of pure Gerstmann syndrome. Interpretation: Our functional neuroimaging findings are not in agreement with Gerstmann's postulate of damage to a common cognitive function underpinning clinical semiology. Our evidence from intact functional neuroanatomy suggests that pure forms of Gerstmann's tetrad do not arise from lesion to a shared cortical substrate but from intraparietal disconnection after damage to a focal region of subcortical white matter.
\end{abstract}

Ann Neurol 2009;66:654-662

In a series of works starting in the 1920 s, Josef Gerstmann described a tetrad of acalculia, finger agnosia, left-right disorientation, and agraphia that he had observed in a few patients. ${ }^{1-3}$ He claimed that this association constituted a syndrome, resulted from damage to the left parietal lobe and expressed a Grundstörung, ie, damage to a common functional denominator that is essential across these four cognitive faculties. Despite heavy attack from cohort-based neuropsychological studies, the first two of Gerstmann's three claims were confirmed by several well-documented modern era single cases with pure Gerstmann syndrome. ${ }^{1,4,5}$ Conceptually, even contemporary reports still interpret Gerstmann's syndrome in terms of a Grundstörung although the flavor of the putative critical cognitive function has changed. ${ }^{1}$ Yet to date there is no direct evidence for a functional association of the four domains that fail in Gerstmann syndrome. While intraoperative electro- stimulation has found a close spatial proximity of the four domains it failed to identify a single site where disruptive stimulation would elicit all four symptoms and also showed that many other cognitive functions are readily disrupted at these parietal sites. ${ }^{6,7}$ We therefore pursued an alternative hypothesis according to which the selective association of the four symptoms that has been reported in pure cases might rather be due to a circumscribed subcortical lesion yielding a disconnection syndrome. ${ }^{8-11}$

We tested this hypothesis by performing in-depth functional and structural neuroimaging in a series of healthy subjects. To avoid spurious overlap from blurring and intersubject brain variability we conducted our experiment at high spatial resolution and sensitivity and analyzed our findings as a series of single cases. In a first step, we recorded brain activation in response to experimental and control conditions that were construed to
From ${ }^{1}$ Institut Nationale de la Santé et de la Recherche Médicale (INSERM), Unité 562, Gif-sur-Yvette, France; ${ }^{2}$ Institute of Cognitive Neuroscience, University College London, London, UK; ${ }^{3} \mathrm{Com}-$ missariat à l'Energie Atomique (CEA), Direction des Sciences du Vivant (DSV), Institut d'Imagerie BioMédicale (I2BM), NeuroSpin, Gif-sur-Yvette, France; and ${ }^{4}$ Université Paris-Sud, Orsay, France.

Address correspondence to Dr Andreas Kleinschmidt, INSERM Unit 562, F91191 Gif-Sur-Yvette, France; E-mail: andreas. kleinschmidt@cea.fr
Additional Supporting Information may be found in the online version of this article.

Potential conflict of interest: Nothing to report.

Received Nov 27, 2008, and in revised form Apr 16, 2009. Accepted for publication May 22, 2009. Published online, in Wiley InterScience (www.interscience.wiley.com). DOI: 10.1002/ana.21776 
identify cortical candidate regions where damage would putatively result in one of the four symptoms of Gerstmann's tetrad, and we then tested for overlap of these four cortical patterns. In a second step, based on diffusion tensor imaging data, we used the functional results to define seeding points for tracking the white-matter connections of the activated cortical zones, and we again examined whether there was an area of overlap between these four groups of white-matter tracts.

\section{Subjects and Methods}

\section{Participants and Imaging Parameters}

Five neurologically healthy right-handed volunteers ( $1 \mathrm{fe}-$ male, 4 male, mean age 21 years) gave written informed consent to participate in the study, which was approved by the local ethics committee. This sample size is adequate for the purpose as well as the means employed in this study, which involved in-depth analyses in a series of single subjects rather than relying on group averaging. ${ }^{12}$ Functional magnetic resonance imaging (fMRI) images were acquired on a Siemens Trio 3T whole-body scanner with a gradient-echo echoplanar imaging sequence $\left(\mathrm{TE}=30 \mathrm{~ms}\right.$, flip angle $=78^{\circ}$, voxel size $=1.5 \times 1.5 \times 2.0 \mathrm{~mm}^{3}$, and no gap); 32 axial slices were acquired every $3 \mathrm{sec}$, covering the parietal lobe, its underlying white matter, and most of prefrontal cortex. Each task sequence consisted of 94 scan volumes for the left/right orientation and finger gnosis tasks, 100 scan volumes for the writing task, and 103 scan volumes for the calculation task. Anatomical images were acquired as $176 \mathrm{~T} 1$-weighted sagittal slices $\left(\right.$ voxel size $=1.0 \times 1.0 \times 1.0 \mathrm{~mm}^{3}, \mathrm{TE}=4.18 \mathrm{~ms}$, $\mathrm{TR}=2.3 \mathrm{sec}$, flip angle $=9^{\circ}$, inversion time $=900 \mathrm{~ms}$, and field-of-view $[\mathrm{FOV}]=256 \mathrm{~mm}$ ). Diffusion-weighted imaging (DWI) used a spin-echo echo-planar technique with $b$ factor $=700 \mathrm{sec} / \mathrm{mm}^{2}(\mathrm{TE}=81 \mathrm{~ms}$ and $\mathrm{TR}=14 \mathrm{sec})$, and 41 diffusion gradient encoding orientations. Sixty interleaved transversal slices covering the whole brain were acquired $\left(\right.$ voxel size $=1.9 \times 1.9 \times 2.0 \mathrm{~mm}^{3}$, and FOV $\left.=240 \mathrm{~mm}\right)$.

\section{Functional Activation Paradigm}

Each of the four tasks - calculation, left-right orientation, finger gnosis, and writing - was studied by contrasting cerebral activations in a related experimental condition with those in a control task that was approximately matched in terms of sensory input, motor output, and nonspecific cognitive aspects (for more details see Fig 1 and the Supporting Information). Briefly, the calculation task involved adding and subtracting sequentially presented numbers interspersed with "+" or "-" symbols. The result was then to be reported by key press when it occurred within a sequence of numbers presented after the calculation epoch. In the control task, numbers were replaced by letters and the task was to report whether or not the letter shown in red at the end of each trial had also been shown in the preceding series. To probe left-right orientation, subjects reported on which side of a manikin drawing, which could be in front or back view, a dot was superimposed. Using the same stimuli, this condition was contrasted with one where subjects reported whether the dot was left or right in their field of view, whatever the orientation of the manikin. For finger gnosis, sub- jects reported by button press those out of a sequence of pictures of a hand where the ring finger was extended. In the control task with the same stimuli, they reported whenever the palm was visible, regardless of the identity of extended and flexed fingers. Finally, to assess graphia, subjects wrote down dictated pseudowords, then this was contrasted with listening to the same words while they only hatched onto paper.

Prior to scanning, subjects underwent a training session with each task until they felt confident. During scanning, we repeated each task in two separate sessions, yielding a total of eight sessions per subject. Each scanning session included three blocks of the experimental condition and three of the control condition, and these six blocks were separated by five baseline epochs of rest, during which participants were asked to fixate on a white central dot on a dark background for 20sec. Experimental and control conditions were done in a counterbalanced alternating order $(\mathrm{ABABAB}$ or $\mathrm{BABABA})$. Each session was preceded by an instruction display describing the two alternating tasks. Once subjects had studied the instruction they pressed a key to start the session. Image acquisition and presentation of behavioral stimuli were synchronized by this latter key press. Using E-Prime 1.1.3 software on a personal computer, stimuli were video-projected onto a screen at which subjects looked via a mirror attached to the head coil.

\section{Functional Imaging Data Analysis}

Functional images were processed with Statistical Parametric Mapping software (SPM5; http://www.fil.ion.ucl.ac.uk/spm/ software/spm5). Following motion correction, normalization to the Montreal Neurological Institute (MNI) template, and slight spatial smoothing $(3 \mathrm{~mm}$ kernel), the effects of experimental and control conditions were modeled as epochs convolved with a canonical hemodynamic response function. Contrasts were generated for each subject for the four domains and in addition separately for repeated sessions of the same task, by testing for greater activation during each experimental task than during its control. Because one of the sessions probing left-right orientation failed for technical reasons in two subjects, these sessions were not considered in the analysis. The cerebral activations in the four domains were analyzed by determining in each subject for each of the domains, the areas showing a significant activation in experimental compared to control conditions $(p<0.001$, uncorrected, at voxel level; $p<0.05$, corrected, at cluster level, corresponding to a 30-voxel cluster extent). We then performed for each subject a conjunction analysis across the four domains (testing conjunction null hypothesis contrastwise at $p<0.01$, uncorrected, at voxel level). In the context of our study, the left parietal lobe was considered the appropriate reference volume to correct for multiple comparisons. We hence applied a small volume correction to voxels included in a mask of the left parietal lobe (from WFU-PickAtlas; http://fmri.wfubmc.edu/cms/software). We considered clusters as significant when they survived a corrected $p$-value of $<0.05$ at the voxel level (family-wise error correction).

\section{Structural Imaging Data Analysis}

Diffusion tensor images were preprocessed and corrected for distortion using BrainVISA software (http://brainvisa.info) ${ }^{13}$ 


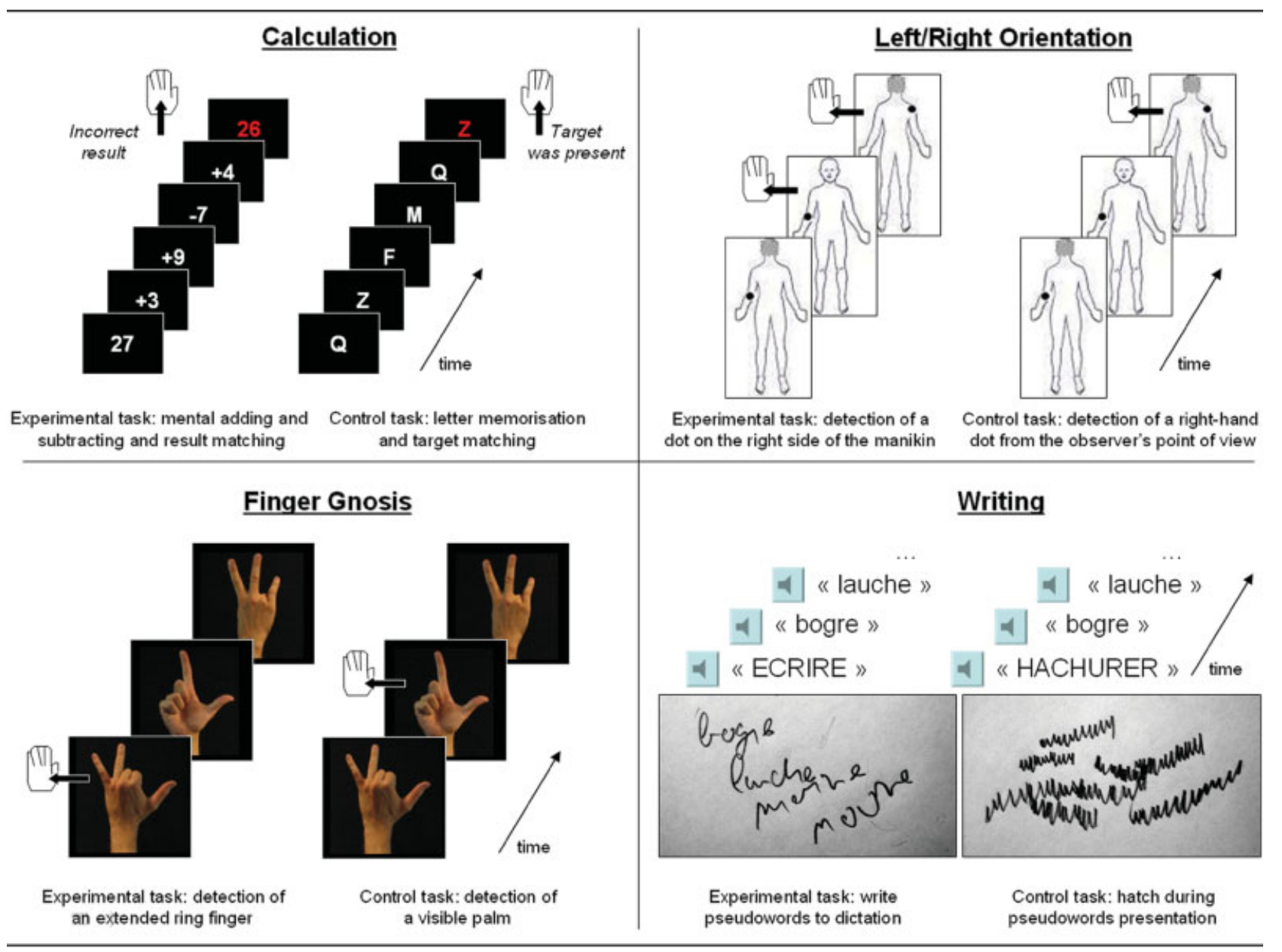

Fig 1. Experimental paradigm addressing the four domains affected in Gerstmann syndrome. Each domain was assessed by contrasting activations in an experimental condition (left side of each quadrant) with those in a control condition (right side) with the related instructions indicated below. In all domains, sensory input was identical or at least matched (calculation) for experimental and control conditions. Where applicable, the number of target occurrences and thus behavioral responses (indicated by hand symbols) were also matched. For the writing and hatching conditions we show examples from the graphic production by one subject during scanning. For more details, see Subjects and Methods as well as Supporting Information.

and visualized using Anatomist software (http://anatomist.info). ${ }^{14}$ White matter tractography was performed from native diffusion-tensor images using BrainVISA. To track fibers from each domain-related individual cortical activation pattern, we extracted seed regions from spatially normalized statistical maps using an automatic procedure (see Supporting Fig 1). Only regions above a threshold of $\mathrm{T}=1.96$ ( $p<0.05$, uncorrected) were considered, to avoid selection of seed regions from noise. The selection of regions was subsequently constrained to the left parietal lobe by masking with a template (defined in WFU-PickAtlas) and unnormalized by applying the individual inverted SPM5 normalization matrix in order to match the native structural image. Fibers were tracked using a deterministic protocol of the FACT type $^{15}$ with anisotropy threshold $=0.2$, maximum angle $=$ $45^{\circ}$, and tracking step $=1 \mathrm{~mm}$.

For each subject, we computed the Boolean intersection of fiber bundle images connected to the cortical activation zones of the four domains. In analogy with the conjunction analysis on the fMRI data and to ensure that for each fiber tract only robust signals would be considered, individual fiber overlap analysis was performed on the four thresholded voxel-wise bundles images at a fiber density value of 30 . This fiber density value is determined by the tractography algorithm we used and expressed in arbitrary units. As a threshold it selects less than the upper $10 \%$ of voxels included into reconstructed tracts. To investigate whether the site of tract overlap was reliable across subjects, we then computed the sum of the normalized intersections (MNI space). Finally, we simulated the impact of a "virtual" lesion at this site by backtracking fibers from the individual parietal white matter site where the fibers that were connected with the four domain-related cortical activations overlapped. The seed region for this analysis was restricted to the overlap found between subjects (to avoid tracking from isolated voxels that were not reproducible over the sample of subjects). We then tracked fibers from this subcortical white-matter seed region using the same parameters as mentioned above. We report the projection of the resulting tracking onto the gray-/white-matter boundary under the cortical surface. To assess whether this disconnection pattern was 


\begin{tabular}{|c|c|c|}
\hline \multicolumn{3}{|c|}{$\begin{array}{l}\text { Table. Behavioral Findings for Each Experimental and Control Task in Three Domains Yielding Quantifiable } \\
\text { Results }\end{array}$} \\
\hline Task & Accuracy & Reaction Time \\
\hline Calculation vs. control & $\begin{array}{l}\text { Experimental task: } 74 \% \text { correct }(\mathrm{SE}= \\
5.68) ; \text { control task: } 98 \% \text { correct }(\mathrm{SE}= \\
1.60) ; T_{(4)}=4.09, \mathrm{MSE}=5.97, p= \\
0.015\end{array}$ & $\begin{array}{l}\text { Experimental task: } 1055 \mathrm{~ms}(\mathrm{SE}=48) \\
\text { control task: } 1011 \mathrm{~ms}(\mathrm{SE}=32) ; T_{(4)}<1\end{array}$ \\
\hline $\mathrm{L} / \mathrm{R}$ orientation vs. control & $\begin{array}{l}\text { Experimental task: } 98 \% \text { correct }(\mathrm{SE}= \\
0.56) ; \text { control task: } 98 \% \text { correct }(\mathrm{SE}= \\
0.47) ; T_{(4)}<1\end{array}$ & $\begin{array}{l}\text { Experimental task: } 607 \mathrm{~ms}(\mathrm{SE}=44) ; \\
\text { control task: } 499 \mathrm{~ms}(\mathrm{SE}=37) ; T_{(4)}=6.57 \text {, } \\
\mathrm{MSE}=16, p=0.003\end{array}$ \\
\hline Finger gnosis vs. control & $\begin{array}{l}\text { Experimental task: } 89 \% \text { correct }(\mathrm{SE}= \\
1.60) ; \text { control task: } 99 \% \text { correct }(\mathrm{SE}= \\
0.37) ; T_{(4)}=7.01, \mathrm{MSE}=1.38, p= \\
0.02\end{array}$ & $\begin{array}{l}\text { Experimental task: } 814 \mathrm{~ms}(\mathrm{SE}=59) \\
\text { control task: } 506 \mathrm{~ms}(\mathrm{SE}=50) ; T_{(4)}=5.97 \text {, } \\
\mathrm{MSE}=52, p=0.004\end{array}$ \\
\hline
\end{tabular}

specific to the precise location of the seed region, we performed additional tracking from three other control seed regions, using spheres in close proximity to the locus of Gerstmann-related fiber overlap $(1 \mathrm{~cm}$ more anterior, $1 \mathrm{~cm}$ more posterior, and $1 \mathrm{~cm}$ more medial, respectively).

\section{Results}

Behavior

For three of four experimental and control tasks we could record and analyze accuracy and reaction time (RT) data that are summarized in the Table. In general, either of these parameters or both indicated greater difficulty for the experimental than for the control condition of each domain. This greater task difficulty in the experimental conditions comes from the fact that we construed experimental and control conditions to share many demands as closely as possible, eg, processing sensory stimuli and generating responses, but that the experimental conditions always contained one further element that was unmatched by the control task and that was specific for one of the four domains that fail in Gerstmann syndrome. For writing and hatching as conditions in the fourth domain, we could not obtain quantitative data but observed that participants complied with the instructions (see representative illustration of performance from one subject during scanning in Fig 1).

\section{Cortical Activation Patterns Mapped by Functional Imaging}

To mirror each of the four elements of Gerstmann syndrome we compared activations between experimental conditions and their respective control conditions. In every subject and session, these domain-related contrasts revealed greater activation in the experimental conditions, usually in several regions of the parietal and frontal lobes and with variable degrees of lateralization to the left hemisphere. Activation in the writing domain was most strongly lateralized to the left hemi- sphere and only two out of five subjects showed any right parietal activation across all four domains. We refrain from describing these activation patterns for each domain in detail because they are beyond the scope of interest of our experiment. For illustration, the domain-related findings in one of the subjects are displayed for both left and right hemispheres in Supporting Fig 2. More importantly and presumably due to the use of a powerful block design, we found the activation maps at high spatial resolution to be very reliable within individual subjects across the two sessions with the same conditions (see axial sections in Supporting Fig 2 for an example). The within-domain robustness and reliability were crucial properties that permitted to assess with confidence the degree of similarity across domains in the following.

The first aim of our experiment was to test for cortical overlap of activations elicited in each of the four domains, calculation, left-right orientation, finger gnosis, and writing. Unthresholded activation maps in parietal cortex from the individual subjects and conditions are displayed in Fig 2 to illustrate the degree of subject-related and domain-related response pattern differences. We tested overlap by computing in each individual subject conjunctions across domains, which can be thought of as a logical AND operation. The resulting maps are presented in Fig 2 for all participating subjects but are visualized at threshold levels of statistical significance that do not correct for the multiple comparisons in the brain volume covered by imaging. To assess the statistical significance of these maps we hence dealt with the multiple comparisons issue in the following, voluntarily permissive manner. As Gerstmann syndrome has been related to dominant parietal lobe damage, we corrected the significance levels of activation foci only for the volume within a mask of the left parietal lobe. Applying a significance threshold of $p<0.05$, corrected, we could not identify any signif- 


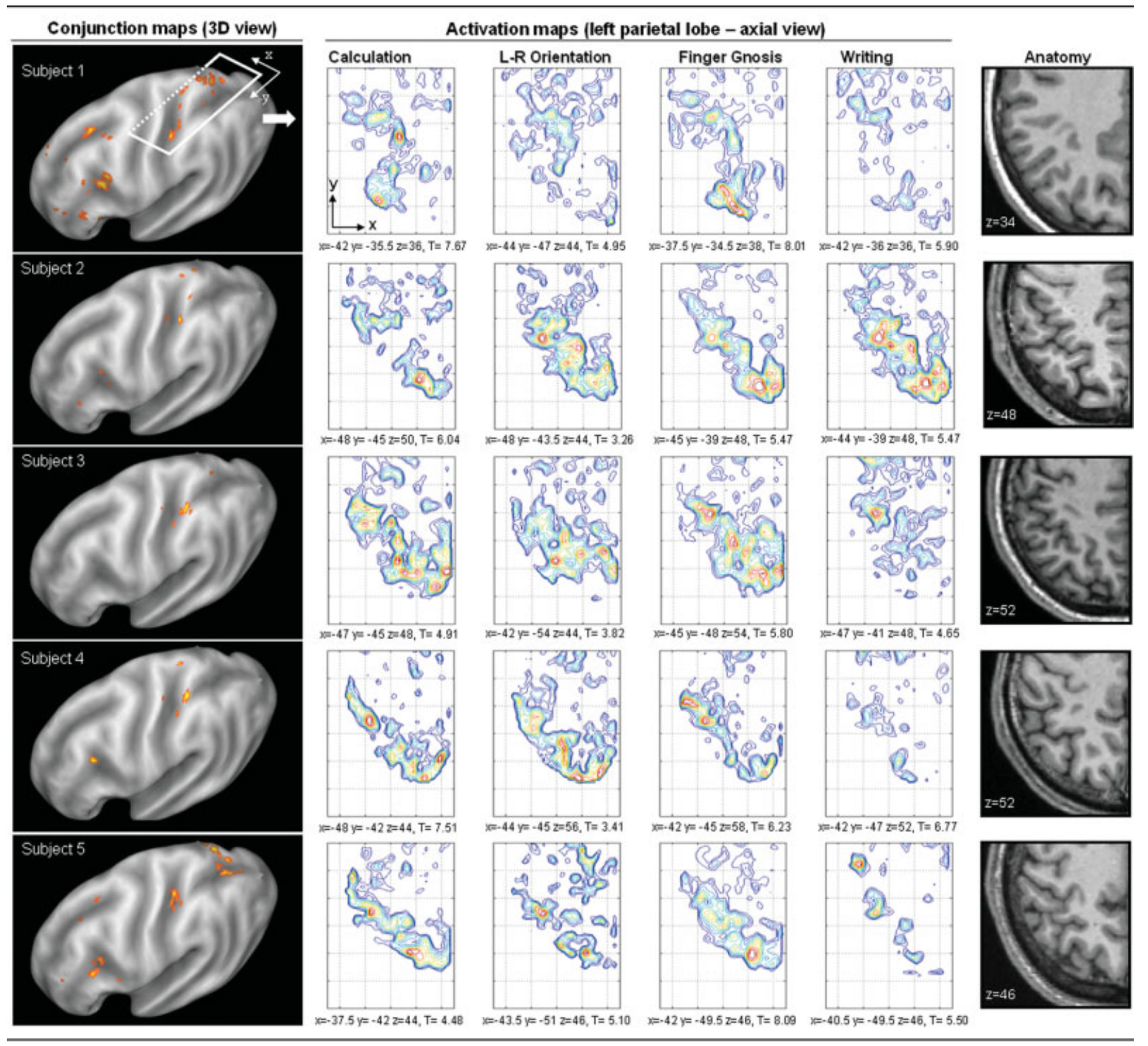

Fig 2. Overview of the functional activation findings in all subjects of the case series. Left-hand column shows surface renderings (3D conjunction maps; using Caret software, http://brainvis.wustl.edu/wiki/index.php/Caret:About ${ }^{27}$ ) of the left hemispheres in all single subjects with superimposed maps of conjunction across the four domains (visualized at a voxelwise $\mathrm{p}<0.01$, uncorrected). None of the conjunction findings displayed here attained statistical significance when correcting for the volume of the left parietal lobe. Middle panels show details of axial sections from activation maps covering the left parietal lobe with corresponding anatomical images from each subject shown in the right-hand column. To visualize for each subject the spatial relation between the left parietal maxima in the activation maps for calculation, orientation, finger and writing tasks, respectively, we depicted on axial planes the statistical parametric map from each domain-related contrast as an isocline of positive T-values. The use of individually adjusted color scaling for the different contrasts permits to illustrate activation patterns as threshold-free landscapes, from lowest (blue) to highest $T$ values (red). Slices were selected such that they included for each domain (column) the response peak closest to the subthreshold anterior intraparietal conjunction sites depicted in the left-hand column. This led to slight z-axis offsets across domains between the selected slices and therefore response peak coordinates are reported along with $T$ values below each panel.

icant parietal conjunction of domain-related activations in any of the subjects. We therefore conclude from the functional neuroimaging data that our experiment did not provide evidence in favor of a specific overlap in parietal activations across all four domains that are affected in Gerstmann syndrome.

\section{Fiber Tracking from Diffusion Tensor Imaging}

The automatic procedure for defining seed regions extracted comparable sets of cortical zones for the four domains (Fig 3A; see also Supporting Fig 1). Tracking starting from these left parietal activation zones identified extensive associated fiber bundles. These domain- 


\section{A}

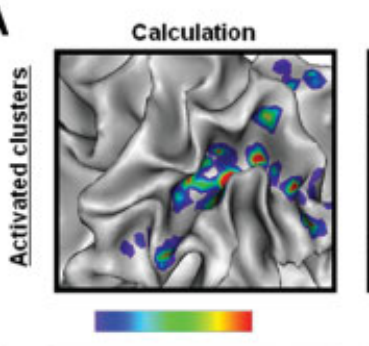

Left-right orientation
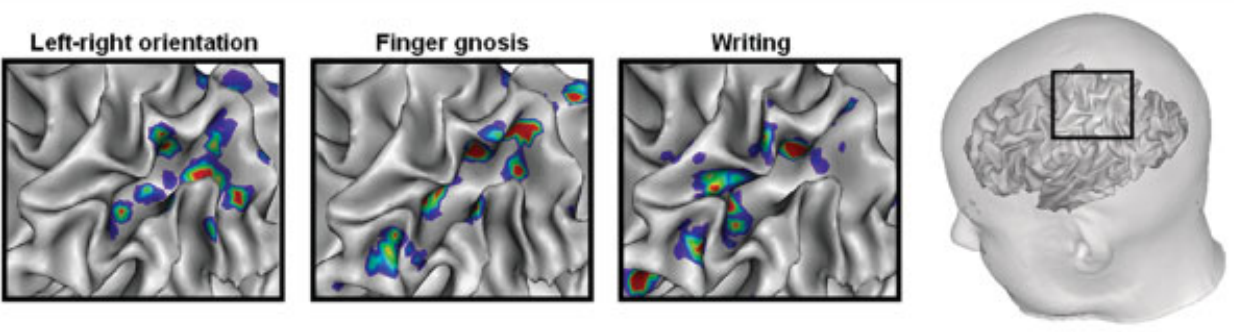

B
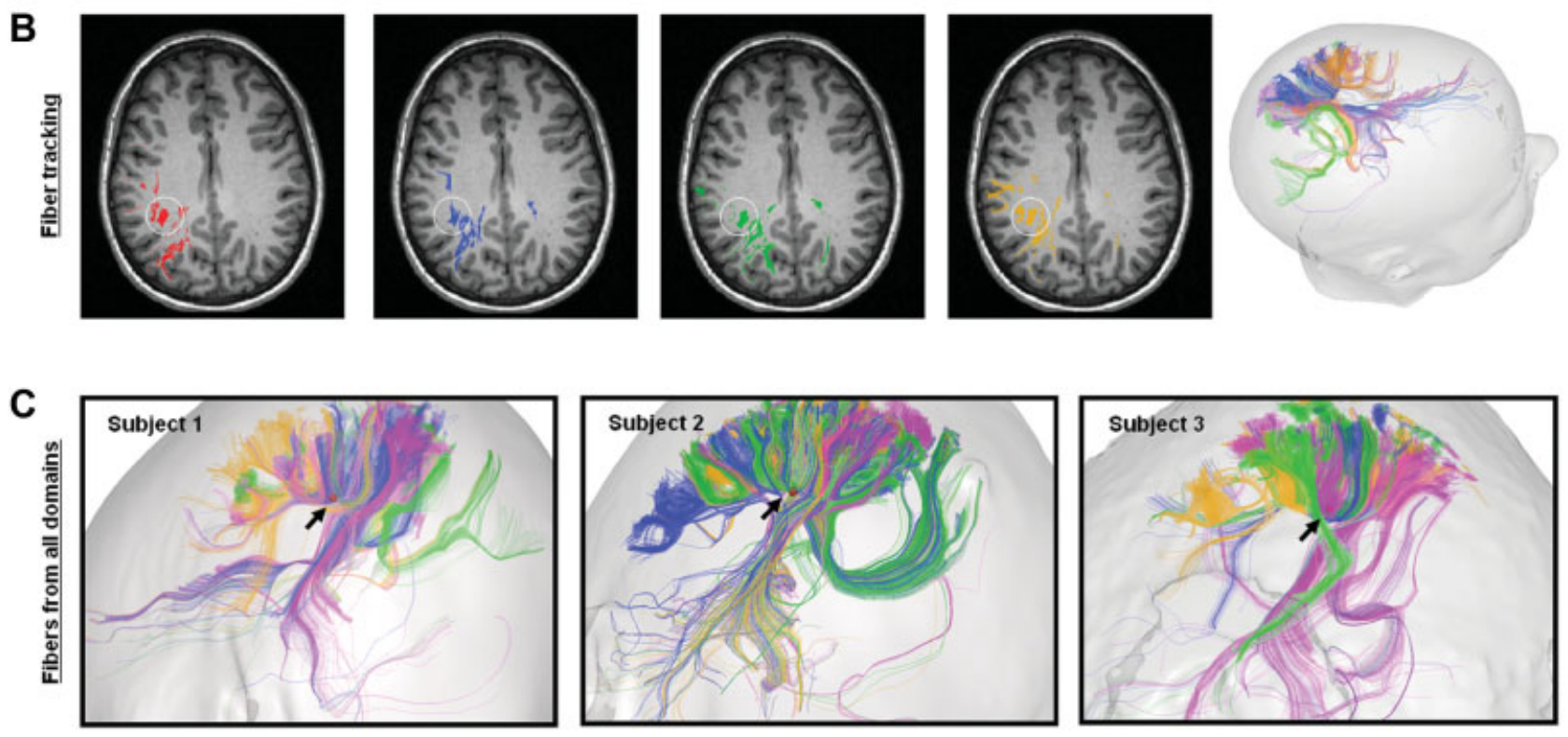

Fig 3. Example of fiber tracking from one subject (subject 1). (A) Functional seed regions used to track fibers related to the four domains, respectively. They are projected onto the subject's left parietal white matter surface with colors representing T-value isoclines. The location of the zoomed detail is indicated on the $3 D$ rendering of the subject's head (right). (B) Axial slice indicating the cross-sectional position of fibers tracts related to the calculation (red), left-right orientation (blue), finger gnosis (green), and writing domain (orange). White circles highlight a white matter region contacted by fibers related to activation patterns from all four domains. Left is on reader's left. The entire four tracings are displayed on a 3D rendering of the subject's head on the right. (C) Results of the tracking procedure for three of the subjects (same color code as in B). Arrows indicate the region of overlap between domains (red dots).

related fiber bundles were intermixed and variable between individuals (Fig 3B, C) but nonetheless also showed a systematic focal overlap (indicated by a white circle in Fig 3B for the four domains in a single subject and by red dots in three-dimensional renderings for three subjects in the Fig 3C). Automatic extraction of fiber bundle overlap across domains confirmed that in all subjects at least one region of white matter, and sometimes but not systematically also more posterior regions of white matter, were contacted by fiber bundles associated with the four different domain-related cortical activations (Fig 4A). The location of reliable overlap relative to anatomical landmarks as the intraparietal sulcus was strikingly similar across subjects. This observation prompted us to probe the degree of spatial reliability between subjects after image normalization into standard stereotactic space. The sum of individual normalized overlap confirmed two closely neighboring sites of maximal intersubject reliability (Fig $4 \mathrm{~B}$, coordinates $-35,-38,35$, and $-35,-45,33$, for $x, y$, and $z$, respectively). Due to fine-grained intersubject variability, however, and thus retrospectively justifying our reluctance to use group averaging procedures in this experiment, results from maximally three out of five subjects overlapped at any given stereotactic coordinates. Nonetheless, the individual results of all subjects fall within the range of the more extensive subcortical lesion in the most recent case report of pure Gerstmann syndrome (Fig 4C). ${ }^{1}$

Next, we asked which pattern of parietal deafferentation or deefferentation a lesion at this white matter location would produce. We simulated the effects of such a lesion by backtracking fibers from a virtual subcortical lesion locus to cortical areas. The bulk of the disconnection effect was produced for cortex of the fundus and walls of the anterior intraparietal sulcus, 
which is not surprising given the proximity of the subcortical overlap zone with these areas. However, other adjacent parietal regions on the convexity were also affected. This pattern was discontinuous and also spared wide regions of the parietal cortex (Fig 5). We then simulated the effects from lesions in neighboring locations by backtracking fibers from adjacent seed regions in left parietal white matter (Fig 5). The related cortical disconnection patterns were strikingly different. It is hence easily conceivable that even subcortical lesions should not readily generate a pure form of Gerstmann syndrome but might produce a varying or overall more extensive profile of functional impairment.

\section{Discussion}

Gerstmann proposed that damage to a single cognitive faculty selectively ties together deficits in four separate functional domains: calculation, writing, finger gnosis, and left-right orientation. Our hypothesis that we would find no overlap of cortical activation across all these domains, and thus no evidence for a functional commonality between them, was motivated by three considerations. First, we are not aware of any cognitive model that defines a single cognitive function which if lost would selectively manifest by the Gerstmann tetrad of symptoms. Second, electrical stimulation during open brain surgery never elicited the full tetrad of Gerstmann symptoms from a single cortical locus. ${ }^{6,7}$ Third, the best documented lesion in a pure case was in subcortical parietal white matter. ${ }^{1}$ We therefore hypothesized that the logic tying together the Gerstmann tetrad is not functional but structural. A lesion of separate but spatially convergent fiber pathways involved in the four domains would then cause this syndrome by way of disconnection.

We addressed these issues by testing in healthy subjects for an overlap of cortical zones that are activated by tasks probing each of the four domains. This approach is grounded in the assumption that cortical activation by a functional challenge can be a useful index in at least identifying candidate areas where lesions might affect that given function. Even at lenient significance thresholds targeting only the left parietal lobe, we could not identify significant cortical overlap in any subject. Contrasting this negative observation, we found in every subject that the white matter fiber tracts associated with the left parietal activation zones for the four different domains overlapped in a subcortical "hot spot" that is compatible with the sparse existing lesion data. ${ }^{1}$ As we applied a rather conservative threshold in the computation of fiber tract overlap, it is possible that we missed further overlap zones for less prominent tracts.

Our findings shed an interesting light on the clinical consequences of damage to the dominant parietal lobe. Neither the constituent symptoms of Gerstmann syn- drome nor lesions to this region of white matter are uncommon, but their selective association in pure Gerstmann syndrome is a seldom clinical event. In cortical degeneration as in Alzheimer's disease, all individual Gerstmann symptoms are readily found but are not more frequently associated with each other than with yet other signs of parietal dysfunction. ${ }^{16}$ Our findings of extended but nonoverlapping parietal activations are in accord with this lack of selective association at the cortical level. Since many more cognitive functions rely on the left parietal cortex it is likely that Gerstmann syndrome from cortical damage would not manifest in a pure form but be associated with other neurological symptoms. ${ }^{17}$ This drowning of Gerstmann symptoms in other deficits as aphasia and/or apraxia has in the past cast doubt on the very existence of this syndrome, especially in light of the ensuing difficulties in neuropsychological testing. ${ }^{18,19}$ Yet, beyond such methodological issues, the hypothesis from our findings would be that any lesion including the circumscribed subcortical site of fiber overlap should generate all four Gerstmann symptoms, thus qualifying it as a disconnection syndrome. This hypothesis could be validated by interrogating in patient samples the coordinates of the fiber overlap site and assessing the related clinical symptoms. However, even purely subcortical lesions will often be larger than the rather restricted fiber convergence zone that we found to associate the four domains. And as adjacent white matter already shows different cortical connection patterns (Fig 5), a pure Gerstmann syndrome remains unlikely.

The last years have seen a renascent interest in disconnection syndromes, largely but not only due to the advent of noninvasive techniques for mapping fiber tract anatomy in the human brain. ${ }^{10}$ Recently, another parietal lobe syndrome, hemineglect, has also been proposed to arise from disconnection. ${ }^{20}$ However, there are a couple of important differences between our proposal and the family of established disconnection syndromes. The latter usually involve damage to a single and long-distance fiber tract and a single clinical manifestation. ${ }^{21-23}$ Conversely, our findings tie together diverse functional domains and involve fiber tracts connecting different regions within a single lobe instead of long-distance connections between, for instance, the parietal and the frontal lobe (see Fig 5). As many lesions involve damage to white matter, our findings hence suggest that the impact of disconnection on clinical deficit might still be largely underestimated.

Another more speculative but interesting hypothesis emerging from our findings regards the relation of postlesional Gerstmann syndrome, to which we have referred so far, and what has been considered a neurodevelopmental disorder yielding a similar clinical phenomenology. Developmental Gerstmann syndrome has been at least as heatedly and controversially debated as 
A Overlap of fibers from the four domains in single subjects

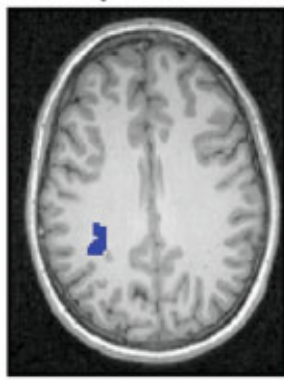

Subject 1

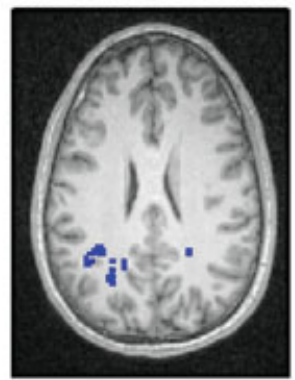

Subject 2

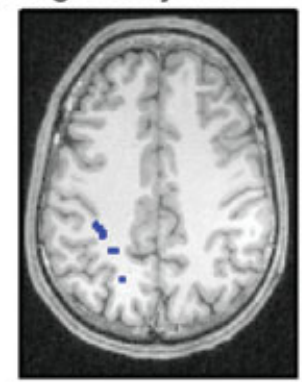

$\underline{\text { Subject } 3}$

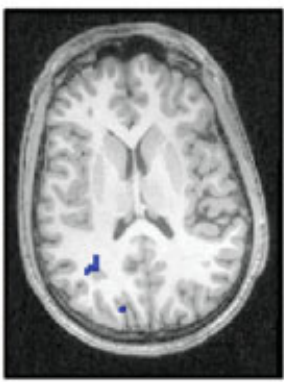

Subject 4

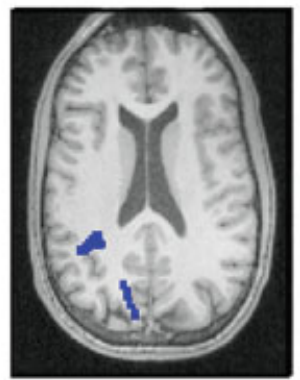

Subject 5
B Sum of spatially normalized single subjects findings
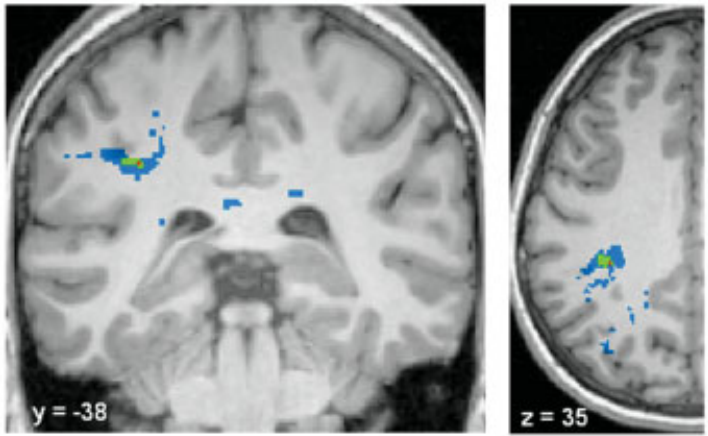

$\square 1$ subject $\square 2$ subjects $\square 3$ subjects

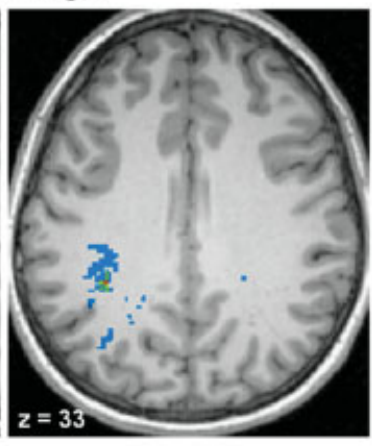

C Example of patient lesion

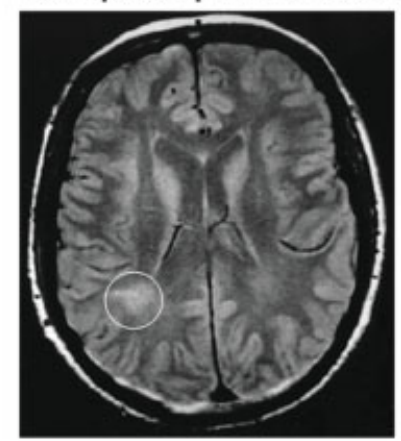

From Mayer et al. (1999)

Fig 4. (A) Overlap of fibers traced for the four domains in the left parietal region of single subjects. (B) Intersection of the five subjects' fiber overlap zones after spatial normalization into standard stereotactic space (left a coronal view; right two axial views). (C) Lesion localization in a patient with pure Gerstmann syndrome illustrated on a T2-weighted axial MRI section (from Mayer et al. $\left.{ }^{1}\right)$. Note spatial congruence of lesion extent with the distribution of probability with which fibers that are related to all four functional domains overlap across subjects. Left is on reader's left.
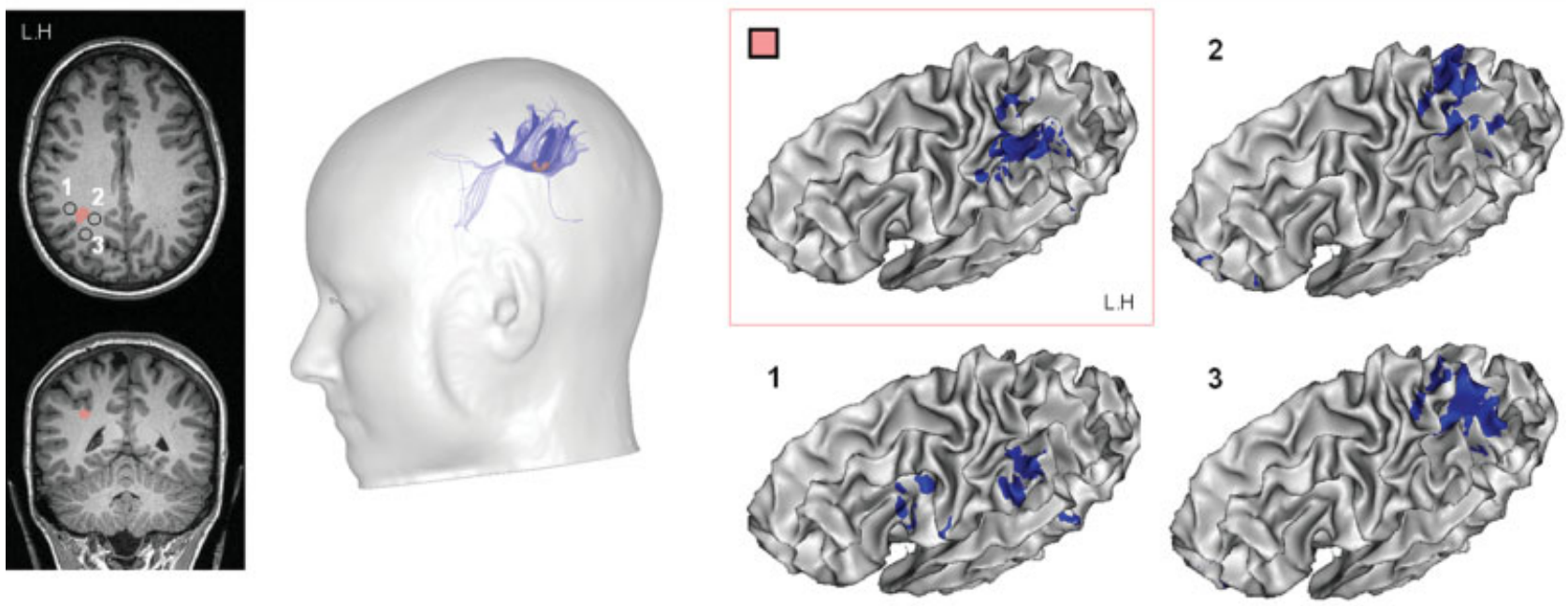

Fig 5. Fiber tracking from seed regions in left parietal white matter. To illustrate putative cortical disconnection effects, we created virtual lesions by taking different subcortical white matter loci as seed regions for fiber tracking. The zone of fiber convergence across the domain-related cortical activations is illustrated in red on the left-hand axial (top) and coronal MRI sections (bottom) and adjacent control regions are numbered. Right-hand panels present the related fiber tracking results (blue) with a threedimensional reconstruction for the seed region of interest (middle column) and renderings of the left hemisphere white-matter surface (tilted view from above, in front and the side) for all seed regions. Small displacements in seed regions yield strikingly different cortical disconnection patterns. 
the postlesional variant. ${ }^{24}$ In recent years, diffusion tensor imaging has associated several other neurodevelopmental disorders such as dyslexia and persistent stuttering with white-matter alterations. ${ }^{25,26}$ In each case, fiber tract disorganization was located in white matter underlying cortical areas that are critical for the impaired functions. Again, our results could therefore serve as a starting point for investigating the relation between structural white-matter integrity and cognitive function in the four domains that are compromised in developmental Gerstmann syndrome.

This work was supported by the Volkswagen Foundation (E.E., S.D., A.K.); and by the European Commission Experienced Researcher Fellowship (MRTN-CT-2003-504927) (E.R.) and Reintegration Grant (MERG-CT-046511) (E.R.).

We thank Laurent Laribière for help with the stimulation setup, Cyril Poupon, and the developers of BrainVisa and Anatomist software.

\section{References}

1. Mayer E, Martory MD, Pegna AJ, et al. A pure case of Gerstmann syndrome with a subangular lesion. Brain 1999;122: 1007-1020.

2. Gerstmann J. Fingeragnosie. Eine umschriebene Störung der Orientierung am eigenen Körper. Wien Klin Wochenschr 1924;40:1010-1012. [German] Finger agnosia. A circumscript disturbance of orientation with respect to the own body.

3. Gerstmann J. Syndrome of finger agnosia, disorientation for right and left, agraphia and acalculia. Arch Neurol Psychiatry 1940;44:398-407.

4. Benton AL. Gerstmann's syndrome. Arch Neurol 1992;49: 445-447.

5. Roeltgen DP, Sevush S, Heilman KM. Pure Gerstmann's syndrome from a focal lesion. Arch Neurol 1983;40:46-47.

6. Morris HH, Lüders H, Lesser RP, et al. Transient neuropsychological abnormalities (including Gerstmann's syndrome) during cortical stimulation. Neurology 1984;34:877-883.

7. Roux FE, Boetto S, Sacko O, et al. Writing, calculating, and finger recognition in the region of the angular gyrus: a cortical stimulation study of Gerstmann syndrome. J Neurosurg 2003; 99:716-727.

8. Geschwind N. Disconnexion syndromes in animals and men. Part I. Brain 1965;88:237-294.

9. Geschwind N. Disconnexion syndromes in animals and men. Part II. Brain 1965;88:585-644.
10. Catani $\mathrm{M}$, fftyche $\mathrm{DH}$. The rises and falls of disconnection syndrome. Brain 2005;12:2224-2239.

11. Catani $M$, Mesulam M. What is a disconnection syndrome? Cortex 2008;44;8:911-913.

12. Friston KJ, Holmes AP, Worsley KJ. How many subjects constitute a study? Neuroimage 1999;10:1-5.

13. Cointepas Y, Mangin J-F, Garnero L, et al. BrainVISA: software platform for visualization and analysis of multi-modality brain data. Neuroimage 2001;6:S98.

14. Rivière D, Mangin JF, Papadopulos-Orfanos D, et al. Automatic recognition of cortical sulci of the human brain using a congregation of neural networks. Med Image Anal 2002;6: 77-92.

15. Mori S, Crain BJ, Chacko VP, van Zijl PC. Three-dimensional tracking of axonal projections in the brain by magnetic resonance imaging. Ann Neurol 1999;45:265-269.

16. Wingard EM, Barrett AM, Crucian GP, et al. The Gerstmann syndrome in Alzheimer disease. J Neurol Neurosurg Psychiatry 2002;72:403-405.

17. Simon O, Mangin JF, Cohen L, et al. Topographical layout of hand, eye, calculation and language-related areas in the human parietal lobe. Neuron 2002;33:475-487.

18. Critchley M. The enigma of Gerstmann's syndrome. Brain 1966;89:183-199.

19. Kinsbourne M, Warrington E. A study of finger agnosia. Brain 1962;85:47-66.

20. Thiebaut de Schotten M, Urbanski M, et al. Direct evidence for a parietal-frontal pathway subserving spatial awareness in humans. Science 2005;309:2226-2228.

21. Epelbaum S, Pinel P, Gaillard R, et al. Pure alexia as a disconnection syndrome: new diffusion imaging evidence for an old concept. Cortex 2008;44:962-974.

22. Catani M, Mesulam M. The arcuate fasciculus and the disconnection theme in language and aphasia: history and current state. Cortex 2008;44:953-961.

23. Heilman KM, Watson RT. The disconnection apraxias. Cortex 2008;44:975-982.

24. Miller CJ, Hynd GW. What ever happened to developmental Gerstmann's syndrome? Links to other pediatric, genetic, and neurodevelopmental syndromes. J Child Neurol 2004;19: 282-289.

25. Klingberg T, Hedehus M, Temple E, et al. Microstructure of temporo-parietal white matter as a basis for reading ability: evidence from diffusion tensor magnetic resonance imaging. Neuron 2000;25:257-259.

26. Sommer M, Koch MA, Paulus W, et al. Disconnection of speech-relevant brain areas in persistent developmental stuttering. Lancet 2002;359:2085-2086.

27. Van Essen DC, Dickinson J, Harwell J, et al. An integrated software system for surface-based analyses of cerebral cortex. J Am Med Inform Assoc 2001;8:443-459. 